\title{
Direct Measurement of Carbon Fusion at Astrophysical En- ergies with Gamma-Particle Coincidences
}

\author{
M. Heine ${ }^{1, *}, G$. Fruet ${ }^{1}, S$. Courtin $^{1,2}$, D.G. Jenkins ${ }^{3}, P$. Adsley $^{4}, A$. Brown $^{3}, R$. Canavan $^{5,6}$,

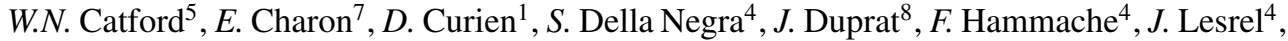 \\ G. Lotay $^{5}$, A. Meyer ${ }^{4}$, E. Monpribat ${ }^{1}, D$. Montanari ${ }^{1,2}, L$. Morris ${ }^{3}, M$. Moukaddam ${ }^{1}, J$.

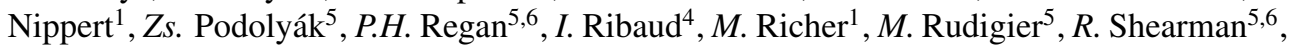 \\ $N$. de Séréville ${ }^{4}$, and $C$. Stodel ${ }^{9}$ \\ ${ }^{1}$ Université de Strasbourg, CNRS, IPHC UMR 7178, F-67000 Strasbourg, France \\ ${ }^{2}$ USIAS/Université de Strasbourg, Strasbourg, F-67083, France \\ ${ }^{3}$ University of York, York, YO10 5DD, UK \\ ${ }^{4}$ IPN, CNRS/IN2P3, Université Paris-Sud, Université Paris-Saclay, F-91406 Orsay Cedex, France \\ ${ }^{5}$ Department of Physics, University of Surrey, Guildford, GU2 7XH, UK \\ ${ }^{6}$ National Physical Laboratory, Teddington, Middlesex, TW110 LW, UK \\ ${ }^{7}$ NIMBE, CEA, CNRS, Université Paris-Saclay CEA Saclay F-91191 Gif sur Yvette, France \\ ${ }^{8}$ CSNSM, Université Paris Sud, UMR 8609-CNRS/IN2P3, F-91405 Orsay, France \\ ${ }^{9}$ GANIL, CEA/DSM-CNRS/IN2P3, Caen, F-14076, France
}

\begin{abstract}
We present ${ }^{12} \mathrm{C}+{ }^{12} \mathrm{C}$ direct fusion measurements with STELLA UKFATIMA, that reach into the region of astrophysics interest relevant to massive stars $\left(M_{\odot} \approx 25\right)$ using self-supporting thin rotating carbon foils [1]. We demonstrate that detecting gammas and light charged particles in coincidence with nanosecond timing is key for effective background reduction achieving reliable measurements in the sub-nanobarn range. We give details about core developments of the detection apparatus as well as the coincidence-analysis procedure of low count statistics. The present data largely follows the phenomenological hindrance interpolation and shows indication for resonant behaviour at the lowest energy explored.
\end{abstract}

\section{Introduction}

Fusion reactions play an essential role in the energy production, the nucleosynthesis of chemical elements and the evolution of massive stars. Among these reactions, carbon burning is a crucial ingredient to understand the late stages of massive stars [2] essentially driven by the ${ }^{12} \mathrm{C}+{ }^{12} \mathrm{C}$ reaction. It presents prominent resonances at energies ranging from a few $\mathrm{MeV} /$ nucleon down to sub-Coulomb barrier energies, possibly due to molecular ${ }^{12} \mathrm{C}-{ }^{12} \mathrm{C}$ configurations of ${ }^{24} \mathrm{Mg}[3,4]$. The possible persistence of these resonances to deep subCoulomb barrier relative energies causes colossal uncertainties of extrapolations of ${ }^{12} \mathrm{C}+{ }^{12} \mathrm{C}$ fusion cross section towards the experimentally challenging region of astrophysics interest with extremely small cross sections. At deep sub-Coulomb barrier energies, fusion hindrance

\footnotetext{
*e-mail: marcel.heine@iphc.cnrs.fr
} 
has been evidenced for heavier systems [5], in present microscopic calculations being interpreted as Pauli blocking of fusion states [6], globally reducing the probability of reaction even further.

\section{Direct Measurements with Thin Targets}

The direct measurement of key fusion reactions at stellar energies offers an unbiased and evident experimental access, but calls for innovative measures for efficient background reduction. In the present approach, with relative energies $E_{\text {rel }}=2.2$ to $5.4 \mathrm{MeV}$, for identifying

$$
{ }^{12} \mathrm{C}+{ }^{12} \mathrm{C} \rightarrow{ }^{20} \mathrm{Ne}^{*}+\alpha \text { and }{ }^{12} \mathrm{C}+{ }^{12} \mathrm{C} \rightarrow{ }^{23} \mathrm{Na}^{*}+\mathrm{p}
$$

reactions by means of detecting the associated de-excitation gammas $\left(\operatorname{LaBr}_{3}(\mathrm{Ce})\right.$ detectors) of light charged evaporated particles (annular silicon detectors), experiments were performed with the STELLA (STELar LAboratory) apparatus [7] in synchronized trigger-less timestamped data recording mode with UK-FATIMA (FAst TIMing Array) [8] at Andromède at IJC Lab, Orsay (France). The $\mathrm{C}^{2+, 3+}$ beam intensity was increased from $30 \mathrm{pnA}$ around the Coulomb barrrier at $6.6 \mathrm{MeV}$ to $2 \mathrm{p} \mu \mathrm{A}$ for the astrophysically relevant region.

The accuracy of data-taking is guaranteed on the one hand by time-walk corrections using simulations of the self activity of the $\mathrm{LaBr}_{3}(\mathrm{Ce})$ detectors [7], that is associated with ${ }^{138} \mathrm{La}$. This background component leads, combined with the decay of members of the ${ }^{227}$ Ac chain, to a counting rate of 1 to $10 \mathrm{~Bq} / \mathrm{cm}^{3}$, and is efficiently removed from the physics spectra by demanding associated charged particles coincidences. On the other hand, the well isolated background from elastic scattering on deuterium is traced in a combined analysis with repeated alpha-source calibrations of the silicon detectors to ensure reliable operation.

The rotating target mechanism supports large diameter $(\sim 5 \mathrm{~cm})$ thin $\left(20\right.$ to $\left.70 \mu \mathrm{g} / \mathrm{cm}^{2}\right)$ carbon foils that spin with up to $1000 \mathrm{rpm}$ for efficient heat dissipation in order to prevent target deterioration. The online target-monitoring routine of comparing the in-beam intensity with off-beam elastic scattering rates was backed by off-line foil-thickness and homogeneity measurements after irradiation confirming that the effect of possible carbon buildup in the beam spot position is prevented by the ultra-high vacuum environment $\left(\approx 10^{-8}\right.$ mbar $)$ and below $1 \%$. In addition, Raman spectrometry analysis of irradiated and non-irradiated areas of selected target foils concludes no significant change of structure of the carbon material under beam exposure.

\section{STELLA UK-FATIMA Fast Timing Coincidence Data}

A particle spectrum in coincidence with a gamma signal is displayed in Figure 1 as a function of the gamma-particle time-difference $\Delta t$, where the $\mathrm{LaBr}_{3}(\mathrm{Ce})$ act as a "start" detector. Evaporation particles populating the $i$-th excited states with alpha emission are indicated in black $\left(\alpha_{i}\right)$ and with proton emission in red $\left(\mathrm{p}_{i}\right)$, respectively, to the left. The synchronization of the $125 \mathrm{MHz}$ STELLA acquisition with the $1 \mathrm{GHz}$ UK-FATIMA system enables timing gates of around $15 \mathrm{~ns}$ and clear separation for exclusive cross section measurements. The timing difference between alphas and protons is caused by different interaction times in the silicon detector substrate. Horizontal bands are caused by the internal background of the $\mathrm{LaBr}_{3}(\mathrm{Ce})$ crystals, that is virtually removed by gating on the associated gamma energy as demonstrated to the right for the $\alpha_{1}$ and $\mathrm{p}_{1}$ transition. Note that all characteristic proton energies with gamma cascades through the first exited states of ${ }^{23} \mathrm{Na}$ populated by $\mathrm{p}_{1}$ are also visible. 

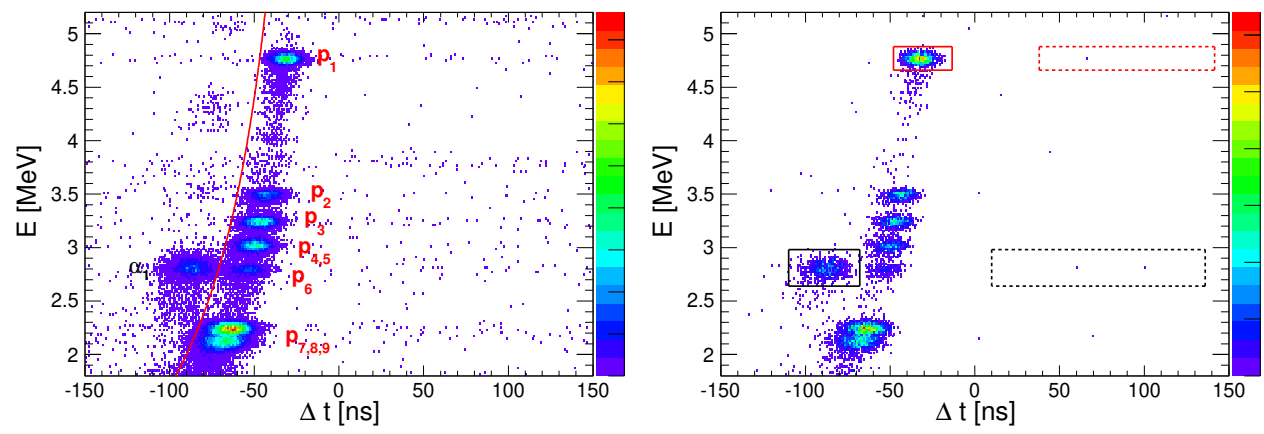

Figure 1. Correlation of charged particle energies with the gamma-particle time-difference. (Left) Evaporation particles with gamma coincidences populating the $i$-th excited states for ${ }^{12} \mathrm{C}+{ }^{12} \mathrm{C} \rightarrow$ ${ }^{20} \mathrm{Ne}^{*}+\alpha_{i}$ are indicated in black and for ${ }^{12} \mathrm{C}+{ }^{12} \mathrm{C} \rightarrow{ }^{23} \mathrm{Na}^{*}+\mathrm{p}_{i}$ in red. (Right) The event selection gates for observations (solid boxes) and background events (dashed boxes) are displayed for the $\alpha_{1}$ and $\mathrm{p}_{1}$ transition, after selection of the associated gamma energy.

The event selection gates for coincident observation (solid boxes) and non-coincident background events (dashed boxes) choosing a wider range for higher statistics are displayed for the $\alpha_{1}$ and $\mathrm{p}_{1}$ transition in Figure 1. The signal $\mu=n-b$ is derived by subtracting the background $b$ from the coincident observation $n$. Towards the lowest relative energies, with exponentially dropping cross sections, a statistical framework adapted to low counting rates was applied [9]. The analysis routine assures that reliable confidence intervals can be attributed e.g. to signals $\mu=0$ with non-zero observation and background measurements, assigning accurate limits depending on the background level.

\section{Carbon Burning $S$-Factors}

The gamma-particle coincidence technique combined with the merit of nanosecond-timing measurements have been used to minimize background contributions for ${ }^{12} \mathrm{C}+{ }^{12} \mathrm{C}$ crosssection determination at the highest precision reached so far in the region of astrophysics interest [10-12]. This has allowed to obtain astrophysical $S$-factors, which are presented in Fruet et al. [1], supplemented by beam and target relevant details of the campaigns as well as the numerical cross-section values.

The results identify three distinct sub-Coulomb energy-regimes: (i) In the relative-energy range $E_{\text {rel }}=4.5$ to $5.5 \mathrm{MeV}$ the cross sections are typically of the order of millibarns. The initial data are in excellent agreement with earlier measurements $[13,14]$ giving confidence in the present methodology and experimental accuracy of measuring the excitation function. (ii) In the deep-sub-barrier regime from $E_{r e l}=2.5$ to $4 \mathrm{MeV}$, where the $S$-factor plateau is located according to the phenomenological hindrance model [15], the Fowler standard extrapolation systematically overestimates the results and hindrance is observed. The present data show remarkable agreement with earlier coincidence measurements utilizing a Germanium-silicon detector array [11] (iii) below $E_{\text {rel }}=2.5 \mathrm{MeV}$, well inside the $25 M_{\odot}$ Gamow window, the $S$-factor rises up that may indicate a change in the fusion mechanism. The situation may indicate either the presence of a resonance and/or might be interpreted as the consequence of the low level density of states in the highly symmetrical compound nucleus ${ }^{24} \mathrm{Mg}$ at these excitation energies [16].

Following the discussion in Fruet et al., the observation of hindrance behavior over a wide energy range necessitates resonant doorway configurations of ${ }^{24} \mathrm{Mg}$ for interpreting the 
measurement at the lowest explored energy. In this picture, fusing is decomposed artificially into two steps, i.e. population of resonances at high excitation energies with subsequent cascading into the rotational bands of ${ }^{24} \mathrm{Mg}$. A more natural explanation might be based on limited phase space in the highly symmetrical even-even nucleus ${ }^{24} \mathrm{Mg}$ with large spacing of narrow levels, so that only a few final states are available for the fusion process to occur.

In the context of quiescent carbon burning in massive stars, the described fusionhindrance bahaviour will lead to elevated carbon-burning ignition temperatures $[17,18]$, as compared to calculations with the Fowler extrapolation. In comparison to this model, less heat is generated by ${ }^{12} \mathrm{C}$ fusion while heat dissipation by neutrinos is basically temperature and density-dependent. Sensitivity studies with plain variations of the $S$-factor across the entire energy range showed furthermore, that the efficiency of the ${ }^{22} \mathrm{Ne}(\alpha, \mathrm{n}){ }^{25} \mathrm{Mg}$ and the ${ }^{13} \mathrm{C}(\alpha$, n) ${ }^{16} \mathrm{O}$ reaction in neutron-seed generation for the $s$-process can change drastically [17]. The present results can be used in further investigations of the advanced burning phases in massive stars, to refine the understanding of their development and the produced neutrino flux of neutrinos. This knowledge will improve the understanding of the probability of type Ia supernovae [18].

\section{Acknowledgments}

STELLA was funded by the University of Strasbourg IdEX program and CNRS Strasbourg, the Andromède facility by ANR-10-EQPX-23. This work was partially supported by the UK grant No. ST/L005743/1, No. ST/P003885/1, and No. ST/P005314/1. P.H.R. and R.S. acknowledge support from the UK Department BEIS. D.G.J. and S.C. acknowledge support from their Fellowships USIAS. J.N. acknowledges support from QMat by grants ANR 10 IDEX 0002 and ANR 20 SFRI 0012.

\section{References}

[1] G. Fruet et al., Physical Review Letters 124, 192701 (2020)

[2] C.E. Rolfs and W.S. Rodney, Cauldrons in the Cosmos (Univ of Chicago Press, 1988)

[3] D. Jenkins and S. Courtin, Journal Physics G 42, 034010 (2015)

[4] Y. Chiba and M. Kimura, Physical Review C 91, 061302(R) (2015)

[5] C.L. Jiang et al., Physical Review Letters 89, 052701 (2002)

[6] C. Simenel et al., Physical Review C 95, 031601(R) (2017)

[7] M. Heine et al., Nuclear Instruments and Methods A 903, 1 (2018)

[8] M. Rudigier et al., Nuclear Instruments and Methods A 969, 163967 (2020)

[9] G.J. Feldman and R.D. Cousins, Phys Rev D 57, 3873 (1998)

[10] T. Spillane et al., Physical Review Letters 98, 122501 (2007)

[11] C.L. Jiang et al., Physical Review C 97, 012801(R) (2018)

[12] W.P. Tan et al., Physical Review Letters 124, 192702 (2020)

[13] H.W. Becker et al., Zeitschrift für Physik A 303, 305 (1981)

[14] E.F. Aguilera et al., Physical Review C 73, 064601 (2006)

[15] G. Montagnoli et al., Physical Review C 97, 024610 (2018)

[16] C.L. Jiang et al., Physical Review Letters 110, 072701 (2013)

[17] M. Pignatari et al., Astrophysical Journal 762, 31 (2013)

[18] O. Straniero et al., Journal of Physics: Conference Series 665, 012008 (2016) 